metachronous recurrence). At a median followup of 36 months after metastectomy, eight patients were still alive, seven of whom were free of recurrence. At 1 year, overall survival was $100 \%$ and disease-free survival was $70 \%$.

The authors recommend that isolated ovarian metastases from colorectal cancer should be treated with bilateral oophorectomy as soon as possible after diagnosis. They have subsequently emphasized that such patients would not need to undergo peritonectomy and perioperative interaperitoneal chemotherapy.

Original article Erroi F et al. (2007) Ovarian metastasis from colorectal cancer: prognostic value of radical oophorectomy. J Surg Oncol [doi: 10.1002/jso.20803]

\section{p95HER2-overexpressing breast tumors have a poor response to trastuzumab}

HER2-overexpressing breast tumors are associated with poor outcome. Even though trastuzumab can bring substantial benefits, $70-80 \%$ of women with HER2-overexpressing breast cancer are unresponsive to monotherapy with this agent. The fact that a subgroup of HER2overexpressing tumors also express p95HER2, a truncated fragment of the HER2 receptor with kinase activity, led to the hypothesis that accumulation of these fragments could be one of the mechanisms behind trastuzumab resistance. A recent study has shown that breast tumors that express p95HER2 are resistant to treatment with the monoclonal antibody trastuzumab.

Scaltriti et al. compared response to trastuzumab and to lapatinib, an inhibitor of HER2, in tumors expressing p95HER2 and those expressing full-length HER2. Treatment of p95HER2-expressing tumor cells with lapatinib inhibited p95HER2 phosphorylation, cell growth and mouse MCF-7p95HER2 xenograft tumor growth, and decreased downstream phosphorylation of Akt and mitogen-activated protein kinases. Treatment with trastuzumab, by contrast, had no effect on any of these parameters. In an additional analysis that utilized a specially developed paraffin-based immunofluorescence assay, only one of nine patients with p95HER2expressing metastatic breast cancer responded to treatment with trastuzumab (partial response), compared with 19 of 37 patients with tumors expressing full-length HER2 (5 complete, 14 partial responses; $P=0.029$ ).
Further stratification of HER2-overexpressing breast tumors into those that do or do not express p95HER2 could, therefore, aid the selection of appropriate anti-HER2 therapy.

Original article Scaltriti M et al. (2007) Expression of p95HER2, a truncated form of the HER2 receptor, and response to anti-HER2 therapies in breast cancer. J Natl Cancer Inst 99: 628-638

\section{Radiation therapy for breast cancer increases the risk of secondary malignancy}

Although radiation therapy (RT) has been shown to reduce 15-year mortality in breast cancer, it is associated with an increased risk of some types of secondary malignancy. To evaluate this risk, Kirova et al. conducted a retrospective review of the records of 16,705 patients treated for nonmetastatic breast cancer (median age 55 years) who were prospectively registered in a French breast cancer database. In total, 13,472 (80.6\%) patients had received RT and 3,233 (19.4\%) had been treated only by mastectomy. Of those who received RT, 2,347 (17.4\%) and 8,596 (63.8\%) had previously undergone mastectomy or lumpectomy, respectively, and in total 5,874 $(43.6 \%)$ had received adjuvant systemic treatment (i.e. chemotherapy, hormonal therapy, or both).

At follow-up (median 10.5 years), 709 patients had developed secondary malignancies, 596 of whom had received RT. In total 35 patients developed sarcoma and 27 of these cases were radiation-induced. There were 58 cases of lung cancer overall, of which 54 were in the RT group; 52 of the 58 patients who developed lung cancer had a history of smoking. There were significant differences between the RT group and the nonRT group in the incidences of secondary lung cancer and of sarcoma (relative risks 3.09 and 7.46, respectively; $P=0.02$ for both). RT was not associated with increased risk of other types of cancer or of contralateral breast cancer.

The authors conclude that RT increases the risk of subsequent lung cancer and sarcoma in patients with nonmetastatic breast cancer, but does not affect the incidence of other types of secondary malignancy.

Original article Kirova YM et al. (2007) Risk of second malignancies after adjuvant radiotherapy for breast cancer: a large-scale, single-institution review. Int J Radiat Oncol Biol Phys 68: 359-363 